\title{
YCG063 inhibits Pseudomonas aeruginosa LPS-induced inflammation in human retinal pigment epithelial cells through the TLR2-mediated AKT/NF-кB pathway and ROS-independent pathways
}

\author{
SUNG HWA PAENG ${ }^{1 *}$, WON SUN PARK ${ }^{2 *}$, WON-KYO JUNG ${ }^{3 *}$, DAE-SUNG LEE ${ }^{4}$, GI-YOUNG KIM ${ }^{5}$, \\ YUNG HYUN CHOI ${ }^{6}$, SU-KIL SEO ${ }^{7}$, WON HEE JANG ${ }^{8}$, JUNG SIK CHOI ${ }^{9}$, \\ YOUNG-MIN LEE ${ }^{9}$, SAEGWANG PARK ${ }^{7}$ and IL-WHAN CHOI ${ }^{7}$
}

${ }^{1}$ Department of Neurosurgery, Busan Paik Hospital, Inje University College of Medicine, Busan; ${ }^{2}$ Department of Physiology, Kangwon National University School of Medicine, Chuncheon, Gangwon; ${ }^{3}$ Department of Biomedical Engineering, and Center for Marine-Integrated Biomedical Technology (BK21 Plus), Pukyong National University, Busan;

${ }^{4}$ Marine Biodiversity Institute of Korea, Seocheon, Chungcheongnam-do; ${ }^{5}$ Laboratory of Immunobiology,

Department of Marine Life Sciences, Jeju National University, Jeju; ${ }^{6}$ Department of Biochemistry,

College of Oriental Medicine, Dongeui University, Busan; Departments of ${ }^{7}$ Microbiology and

${ }^{8}$ Biochemistry, Inje University College of Medicine; ${ }^{9}$ Department of Internal Medicine,

Busan Paik Hospital, Inje University College of Medicine, Busan, Republic of Korea

Received November 12, 2014; Accepted June 22, 2015

DOI: $10.3892 /$ ijmm.2015.2266

\begin{abstract}
YCG063 is known as an inhibitor of reactive oxygen species (ROS); however, its intracellular mechanisms of action remain poorly understood. In the present study, we investigated the effects of YCG063 on the inflammatory response of Pseudomonas aeruginosa lipopolysaccharide (PA-LPS)-stimulated human retinal pigment epithelial cells (RPE cells). Human adult RPE cells (ARPE-19) were stimulated with PA-LPS. We then investigated the LPS-induced expression of several inflammatory mediators, such as interleukin (IL)-6, IL-8, monocyte chemoattractant protein-1 (MCP-1) and intracellular adhesion molecule-1 (ICAM-1) in the ARPE-19 cells. We performed an enzyme-linked immunosorbent assay (ELISA), western
\end{abstract}

Correspondence to: Professor Il-Whan Choi or Professor Saegwang Park, Department of Microbiology, Inje University College of Medicine, Bokji-Ro 75, Busanjin-gu, Busan 614-735, Republic of Korea

E-mail: cihima@inje.ac.kr

E-mail: micpsg@inje.ac.kr

"Contributed equally

Abbreviations: PA-LPS, Pseudomonas aeruginosa lipopolysaccharide; TLR, Toll-like receptor; ROS, reactive oxygen species; RPE cells, retinal pigment epithelial cells; MCP-1, monocyte chemoattractant protein-1; ICAM-1, intracellular adhesion molecule-1

Key words: Pseudomonas aeruginosa, endophthalmitis, retinal pigment epithelium, reactive oxygen species, Toll-like receptor blot analysis, electrophoretic mobility shift assay (EMSA) and fluorescence-activated cell sorting (FACS) to elucidate the mechanisms involved in the anti-inflammatory effects of YCG063 in the $P A$-LPS-stimulated cells. The results revealed that treatment with YCG063 significantly inhibited the levels of IL-6, IL-8, MCP-1 and ICAM-1 in the PA-LPS-stimulated ARPE-19 cells. YCG063 also markedly inhibited the phosphorylation of AKT in the $P A$-LPS-stimulated cells. In addition, the activation of nuclear factor- $\kappa \mathrm{B}(\mathrm{NF}-\kappa \mathrm{B})$ was also attenuated following treatment with YCG063. ROS were not generated in the PA-LPS-stimulated cells. In conclusion, our data indicate that YCG063 may prove to be a potential protective agent against inflammation, possibly through the downregulation of Toll-like receptor 2 (TLR2) and the AKT-dependent NF- $\kappa \mathrm{B}$ activation pathway in PA-LPSstimulated ARPE-19 cells. Furthermore, this anti-inflammatory activity occurred through ROS-independent signaling pathways.

\section{Introduction}

Endophthalmitis is an uncommon intraocular inflammatory condition, but it may result in partial or complete vision loss. It may occur as a complication of intraocular surgery or as a result of non-surgical trauma or systemic infection (1). Bacterial endophthalmitis is an infection of the intraocular cavities that usually occurs following the introduction of microbial organisms into the eye (2). During a bacterial infection, irreversible damage to the photoreceptor cells of the retina frequently occurs (3). Among the bacterial pathogens, Pseudomonas aeruginosa $(P A)$, a Gram-negative rod rod-shaped organism, is frequently a cause of nosocomial infections (4). $P A$ can cause ocular infections, such as keratitis and endophthalmitis (5). 
Lipopolysaccharides (LPS), components of Gram-negative bacteria, lead to the activation of immune cells, resulting in the release of pro-inflammatory mediators (6). Retinal pigment epithelial cells (RPE cells) secrete pro-inflammatory mediators within the eye when stimulated with infectious agents, such as Gram-negative bacteria (7). The retinal pigment epithelium (RPE) is a monolayer of pigmented cells originating from the neural ectoderm. The RPE lies between the photoreceptor cell layer of the neural retina and Bruch's membrane and choroid and contains blood vessels that nourish the retina (8). The major function of the RPE is the phagocytosis of photoreceptor segments, converting light impulses into vision and it is involved in the pathology of a variety of retinal diseases, such as age-related macular degeneration (AMD) and diabetic retinopathy (9). The RPE cells play an important role in the ocular immune system.

Inflammatory mediators play a critical role in orchestrating cellular infiltration to the site of infection. Increased cellular infiltration is followed by severe retinal damage. Interleukin (IL)-6 is a pleiotropic cytokine with a wide spectrum of biological activities in a broad variety of ocular inflammatory diseases and wound healing processes (10-12). IL-6 is an important mediator in a variety of diseases, including inflammatory, autoimmune and malignant diseases. The local production of IL- 6 by resident cells and infiltrating inflammatory cells has been detected during a variety of inflammatory ocular conditions $(13,14)$. IL-6 is involved in the recruitment of polymorphonuclear leukocytes (PMNs) to the site of inflammation by upregulating the expression of intercellular adhesion molecule-1 (ICAM-1), a key molecule involved in the migration of neutrophils (15-17). ICAM-1 is important for the transendothelial migration of leukocytes through blood vessels and the retention of leukocytes in the inflamed tissue by means of firm attachment to the extracellular matrix (18). Monocyte chemotactic protein-1 (MCP-1), a member of the $\mathrm{C}-\mathrm{C}$ subfamily of chemokines, may elicit its inflammatory effect, leading to leukocyte accumulation and activation in areas of injury and inflammation under pathological and physiological conditions (19-21). IL-8, a member of the $\mathrm{C}-\mathrm{X}-\mathrm{C}$ chemokines, is an important mediator of various acute and chronic infectious conditions that attract and activate neutrophils and eosinophils (22).

Damage to the delicate tissues of the retina occurs due to oxidative stress (23). YCG063 (Fig. 1), a novel anti-angiogenic small molecule, inhibits reactive oxygen species (ROS) production (24); however, its anti-inflammatory activity in response to LPS has not previously been identified. In the present study, we examined cultured human RPE cells for the production of IL-6, IL-8, ICAM-1 and MCP and the signaling pathways involved in response to stimulation by $P A$-LPS. Additionally, we evaluated the effects of YCG063 on these inflammatory responses.

\section{Materials and methods}

Reagents. PA-LPS was purchased from Sigma Chemical Co. (St. Louis, MO, USA). YCG063 was obtained from Millipore (Billerica, MA, USA). LY294002 (an inhibitor of AKT) was purchased from Calbiochem (La Jolla, CA, USA). Antibodies against p65 (Cat. no. 14-6731) and Toll-like receptor (TLR)2 (Cat. no. 16-9024-83; clone T2.5) were obtained from eBioscience (San Diego, CA, USA). Antibodies against phosphorylated (p)-extracellular signal-regulated protein kinase (ERK)1/2

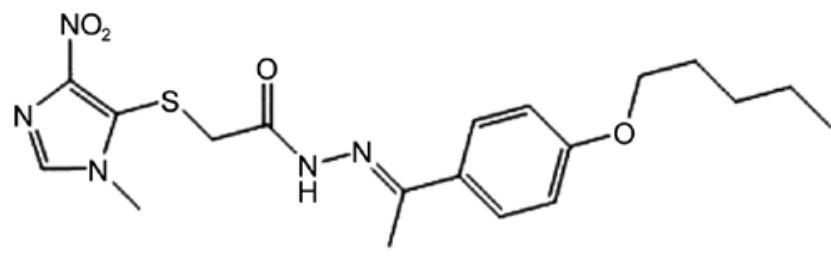

Figure 1. Chemical structure of YCG063.

(Cat. no. 9106), AKT (Cat. no. 9272), p-AKT (Cat. no. 4058) and p-p38 MAPK (Cat. no. 9211) were purchased from Cell Signaling Technology (Beverly, MA, USA). Antibodies against ERK1/2 (Cat. no. sc-94) and p38 MAPK (Cat. no. sc-535) were purchased from Santa Cruz Biotechnology, Inc. (Santa Cruz, CA, USA). 2',7'-Dichlorofluorescein diacetate (DCFDA) was purchased from Invitrogen (Carlsbad, CA, USA). MitoSOX and MitoPY1 were purchased from Tocris Bioscience (Bristol, UK). BAY 11-7082 and parthenolide [nuclear factor- $\kappa \mathrm{B}(\mathrm{NF}-\kappa \mathrm{B})$ inhibitors] were purchased from Santa Cruz Biotechnology, Inc. Nitrocellulose membranes and an enhanced chemiluminescence (ECL) kit were obtained from Amersham Pharmacia Biotech (Uppsala, Sweden). Phycoerythrin (PE)-conjugated anti-human TLR2 (clone TL2.1) and TLR4 (clone HTA125) monoclonal antibodies or isotope controls were obtained from BD Biosciences (San Jose, CA, USA).

ARPE-19 cell culture. The ARPE-19 cells were obtained from the American Type Culture Collection (ATCC, Manassas, VA, USA) and cultured in Dulbecco's modified Eagle's medium (DMEM)/F12 medium supplemented with $10 \%$ fetal bovine serum plus a $100 \mathrm{IU} / \mathrm{ml}$ penicillin and $100 \mu \mathrm{g} / \mathrm{ml}$ streptomycin mixture (Gibco/BRL, Gaithersburg, MD, USA) in a humidified atmosphere $\left(5 \% \mathrm{CO}_{2}\right)$ at $37^{\circ} \mathrm{C}$. The ARPE-19 cells were trypsinized, seeded in 10-cm diameter dishes, and incubated overnight until attachment.

Cell viability assay. The viability of the ARPE-19 cells was determined using the cell counting kit-8 (CCK-8) according to the manufacturer's instructions (Dojindo Laboratories, Kumamoto, Japan). Briefly, the cells were seeded in triplicate at a density of $1 \times 10^{4}$ cells/well into 96-well culture plates and allowed to attach overnight. The cells were stimulated with the indicated concentrations of PA-LPS $(0.5,1,5,10,20$ and $30 \mu \mathrm{g} /$ $\mathrm{ml})$ for $24 \mathrm{~h}$. The medium was then replaced with $100 \mu \mathrm{l}$ of DMEM/F12 medium containing 5,10 or $50 \mu \mathrm{M}$ YCG063. The plates were incubated for $24 \mathrm{~h}$, and $10 \mu \mathrm{l}$ of CCK-8 reagent was added to each well. Following incubation for a further $2 \mathrm{~h}$ at $37^{\circ} \mathrm{C}$, the plates were read at $450 \mathrm{~nm}$ using a microplate reader (Model EL800; Bio-Tek Instruments, Winooski, VT, USA).

Enzyme-linked immunosorbent assay (ELISA). The levels of cytokines in the cell culture medium were assessed by ELISA. The cells were treated with various concentrations of YCG063, BAY 11-7082 and parthenolide for $2 \mathrm{~h}$ prior to $P A$-LPS stimulation. Following incubation for $24 \mathrm{~h}$, the culture supernatants were collected, and the levels of inflammatory mediators were measured. ELISA kits purchased from BioLegend (San Diego, CA, USA) were used to measure the levels of IL-6, IL-8 and MCP-1, and a kit obtained from R\&D Systems (Minneapolis, 

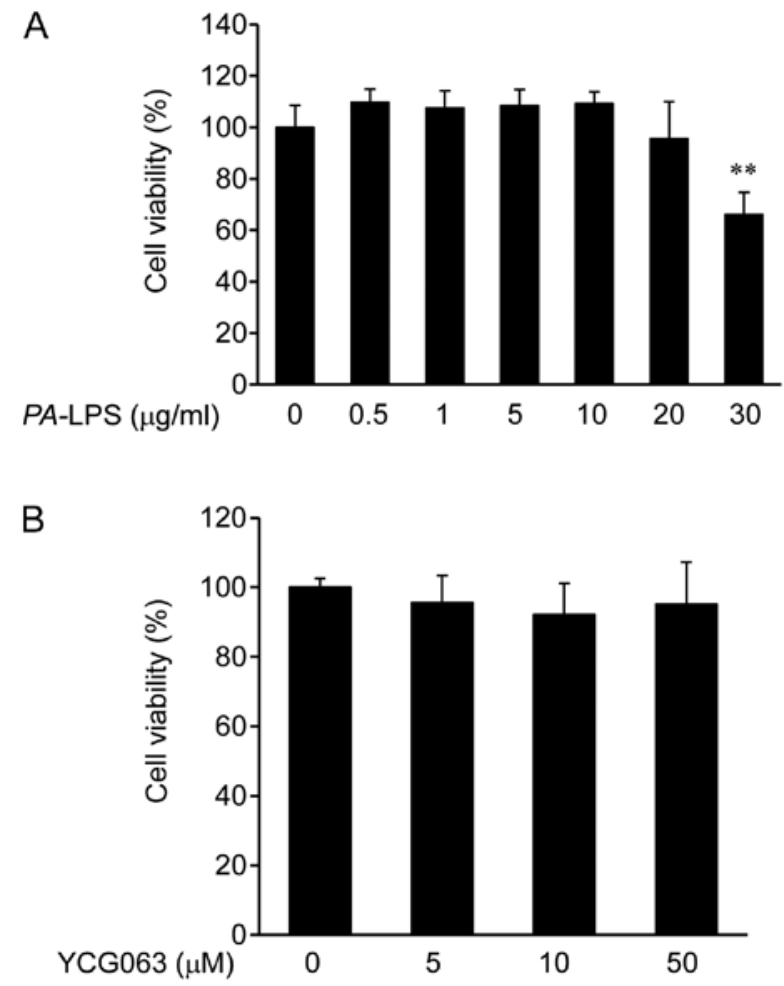

Figure 2. Viability of human adult retinal pigment epithelial cells (ARPE-19 cells). (A) Cells were stimulated with the indicated concentrations of Pseudomonas aeruginosa lipopolysaccharide (PA-LPS; 0.5, 1, 5, 10, 20 and $30 \mu \mathrm{g} / \mathrm{ml}$ ) for $24 \mathrm{~h}$. (B) Cells were treated with the indicated concentrations of YCG063 $(0,5,10$ or $50 \mu \mathrm{M})$ for $24 \mathrm{~h}$, and cell viability was determined. Cell viability was assessed using the cell counting kit-8 (CCK-8) method, and the results are expressed as the percentage of surviving cells over the control cells (no addition of YCG063). Each value indicates the mean \pm SD and is representative of the results obtained from 3 independent experiments. ${ }^{* *} \mathrm{p}<0.01$ compared with the untreated controls.

MN, USA) was used to measure the ICAM-1 levels. The absorbance at $450 \mathrm{~nm}$ was measured using a microplate reader (Model EL800; Bio-Tek Instruments).

Western blot analysis. The ARPE-19 cells were pre-treated with YCG063 and LY294002 for $2 \mathrm{~h}$ prior to stimulation with PA-LPS. The ARPE-19 cells were washed 3 times with phosphate-buffered saline (PBS) and lysed with lysis buffer (Mammalian Cell-PE LB, G-Biosciences, St. Louis, MO, USA). Equal amounts of protein were separated on $10 \%$ sodium dodecyl sulfate (SDS)-polyacrylamide minigels and transferred onto nitrocellulose transfer membranes. Follownig incubation with the appropriate primary antibody (ERK, p-ERK, p38, p-38, AKT, p-AKT and p65), the membranes were incubated for $1 \mathrm{~h}$ at room temperature with a secondary antibody conjugated to horseradish peroxidase [goat anti-rabbit IgG (Cat. no. 31460; Pierce, Rockford, IL, USA), goat anti-mouse IgG (Cat. no. sc-2031; Santa Cruz Biotechnology, Inc.)]. Following 3 washes in Tris-buffered saline Tween-20 (TBST), the immunoreactive bands were visualized using the ECL detection system (Pierce).

Preparation of nuclear extracts and electrophoretic mobility shift assay (EMSA). Nuclear extracts were prepared using the NE-PER nuclear extraction reagent (Pierce). As a probe for the gel retardation assay, an oligonucleotide containing the immunoglobulin $\kappa$-chain binding site $\left(\kappa \mathrm{B}, 5^{\prime}\right.$-GATCTCAGA GGGGACTTTCCGAGAGA-3') was synthesized. A nonradioactive method in which the 3 ' end of the probe was labeled with biotin was used in these experiments (Pierce). The binding reactions contained $5 \mu \mathrm{g}$ of nuclear extract protein, buffer (10 mM Tris, pH 7.5, $50 \mathrm{mM} \mathrm{KCl,} 5 \mathrm{mM} \mathrm{MgCl}_{2}, 1 \mathrm{mM}$ dithiothreitol, $0.05 \%$ Nonidet P-40 and $2.5 \%$ glycerol), $50 \mathrm{ng}$ of poly (dI-dC) and $20 \mathrm{fM}$ of the biotin-labeled DNA. The reactions were incubated for $20 \mathrm{~min}$ at room temperature in a final volume of $20 \mu \mathrm{l}$. The competition reactions were conducted by adding a 100 -fold excess of unlabeled p $65 \mathrm{NF}-\kappa \mathrm{B}$ to the reaction mixture. The mixture was then separated by electrophoresis on a $5 \%$ polyacrylamide gel in $0.5 \mathrm{X}$ Tris-borate buffer and transferred onto nylon membranes. The biotinlabeled DNA was detected using a LightShift chemiluminescent EMSA kit (Pierce).

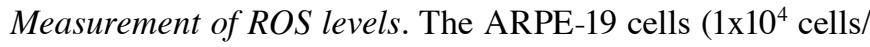
well) were seeded in 96-well plates in a humidified atmosphere containing $5 \% \mathrm{CO}_{2}$ at $37^{\circ} \mathrm{C}$ for $16 \mathrm{~h}$. Following $16 \mathrm{~h}$ of incubation, the cells were treated with $10 \mu \mathrm{g} / \mathrm{ml} P A$-LPS and further incubated for $5 \mathrm{~h}$. For the intracellular ROS assay, the cells were incubated with $10 \mu \mathrm{M}$ DCFDA for $30 \mathrm{~min}$. The cells were then fixed with an equal volume of $4 \%$ formaldehyde and measured immediately. The intracellular ROS levels were measured using a fluoresence microplate reader (SpetraMax M2; Molecular Devices, Sunnyvale, CA, USA) at an excitation wavelength of $492 \mathrm{~nm}$ and an emission wavelength of $515 \mathrm{~nm}$. For the mitochondrial ROS assay, the cells $\left(1 \mathrm{ml}, 1 \times 10^{5}\right.$ cells $\left./ \mathrm{ml}\right)$ were incubated with $5 \mu \mathrm{M}$ MitoSOX and MitoPY1 for $30 \mathrm{~min}$. Subsequently, the cells were washed with PBS and examined immediately. The levels of mitochondrial ROS were measured using FACSort and CellQuest Pro Software (BD Biosciences).

Flow cytometric analysis. The cells were stained with PE-conjugated anti-human TLR2 monoclonal antibody (mAb; clone TL2.1) and PE-conjugated anti-human TLR4 mAb (clone HTA125) and analyzed using FACSort and Cell QuestPro software (BD Biosciences).

Statistical analysis. Data values represent the means \pm standard deviation (SD). To analyze the data produced from the experiments with 2 independent variables, one-way analysis of variance (ANOVA) was performed using GraphPad Prism software (GraphPad Software, La Jolla, CA, USA). A value of $p<0.05$ was considered to indicate a statistically significant difference.

\section{Results}

Effects of PA-LPS and YCG063 on the viability of ARPE-19 cells. Initially, we examined the viability of the ARPE-19 cells stimulated with PA-LPS $(0.5,1,5,10,20$ and $30 \mu \mathrm{g} / \mathrm{ml})$ for $24 \mathrm{~h}$ and of those treated with YCG063 (DMSO, 5, 10 and $50 \mu \mathrm{M}$ ) by CCK-8 assay. No significant cytotoxicity was observed following stimulation with PA-LPS at the lower doses; however, cell viability significnatly decreased following stimulation with $P A-L P S$ at $30 \mu \mathrm{g} / \mathrm{ml}$ (Fig. 2A). There was no significant cytotoxicity observed to the ARPE-19 cells following treatment with YCG063 at doses up to $50 \mu \mathrm{M}$ (Fig. 2B). Based on these results, 

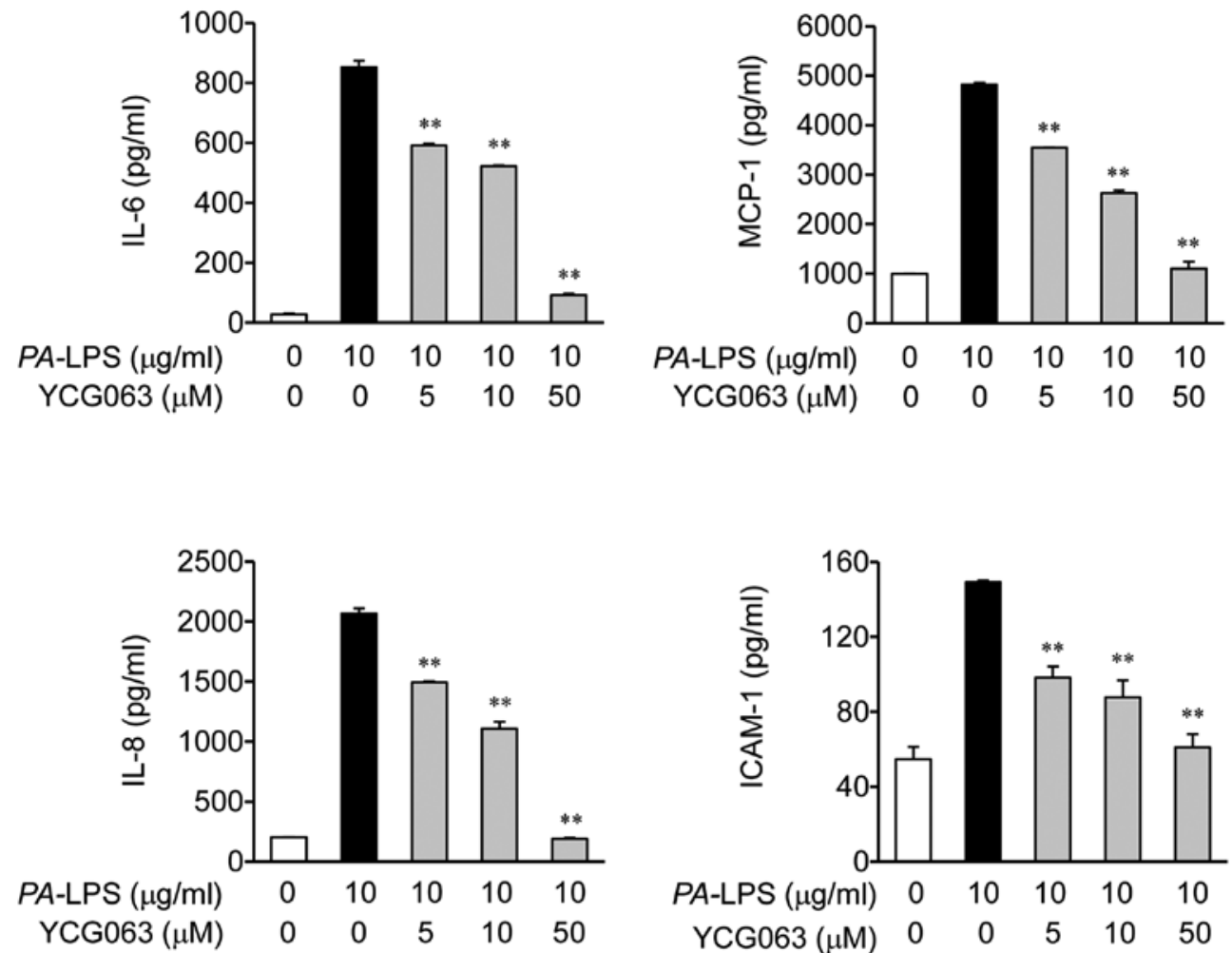

Figure 3. Effect of YCG063 on the production of interleukin (IL)-6), IL-8, monocyte chemoattractant protein-1 (MCP-1) and intracellular adhesion molecule-1 (ICAM-1) in Pseudomonas aeruginosa lipopolysaccharide (PA-LPS)-stimulated human adult retinal pigment epithelial cells (ARPE-19 cells). The protein levels of IL-6, IL-8, MCP-1 and ICAM-1 were determined by ELISA. The cells were treated with YCG063 (5, 10 and $50 \mu \mathrm{M}$ ) for $2 \mathrm{~h}$ prior to LPS stimulation for $24 \mathrm{~h}$. Each bar represents the mean \pm SD from 3 independent experiments. ${ }^{* *}$ p $<0.01$ compared with LPS-stimulated values.

a concentration range of $P A$-LPS of $10 \mu \mathrm{g} / \mathrm{ml}$ and of YCG063 of 5 to $50 \mu \mathrm{M}$ was selected for use in the subsequent experiments.

Effects of YCG063 on the expression of IL-6, IL-8, MCP-1, and ICAM-1 in PA-LPS-stimulated ARPE-19 cells. The levels of IL-6, IL-8, MCP-1 and ICAM-1 considerably increased following stimulation of the ARPE-19 cells with $P A$-LPS (Fig. 3). To evaluate the effects of YCG063 on the protein expression of IL-6, IL-8, MCP-1 and ICAM-1 in the ARPE-19 cells, we treated the cells with YCG063 (5, 10 and $50 \mu \mathrm{M})$ prior to stimulation with $P A$-LPS $(10 \mu \mathrm{g} / \mathrm{ml})$. Treatment with YCG063 suppressed the PA-LPS-induced production of the IL-6, IL-8, MCP-1 and ICAM-1 proteins (Fig. 3).

Expression of TLR2 in PA-LPS-stimulated ARPE-19 cells. To determine whether TLRs are involved in the $P A$-LPS-stimulated inflammatory responses in ARPE-19 cells, the cells were treated with LPS $(10 \mu \mathrm{g} / \mathrm{ml})$ for $12 \mathrm{~h}$. The cells were then stained with PE-conjugated TLR2 and TLR4, and their expression levels were measured by flow cytometry (Fig. 4). The unstimulated cells did not express TLR2, whereas upon stimulation with LPS, the protein expression of TLR2 was upregulated (Fig. 4). However, TLR4 expression was not found to be upregulated during PA-LPS stimulation (Fig. 4). Therefore, as indicatd by the results of flow cytometry, only TLR2 is involved in the $P A$-LPS-induced inflammatory responses of ARPE-19 cells.

Effects of TLR2 antibody on the expression of IL-6, IL-8, MCP-1 and ICAM-1 in PA-LPS-stimulated ARPE-19 cells.
Based on the results shown in Fig. 4, we investigated whether the TLR2 antibody can suppress PA-LPS-induced inflammation (Fig. 5). As expected, the TLR2 blocking antibody (T2.5) inhibited the LPS-induced expression of inflammatory mediators, including the expression of IL-6, IL-8, MCP-1 and ICAM-1 in the ARPE-19 cells, as assessed by ELISA (Fig. 5).

Effects of YCG063 on the activation of the MAPK and AKT signaling pathways in LPS-stimulated ARPE-19 cells. To elucidate the mechanisms underlying the effects of YCG063 on the expression of inflammatory mediators, we examined the activation of MAPKs and AKT by western blot analysis. Stimulation of the ARPE-19 cells with PA-LPS resulted in an increase in the levels of phosphorylated (p-)AKT and p-ERK, but not in those of p-p38. Pre-treatment with YCG063 (10 or $50 \mu \mathrm{M}$ ) for $2 \mathrm{~h}$ attenuated the phosphorylation of AKT induced by a $30-\mathrm{min}$ incubation with $10 \mu \mathrm{g} / \mathrm{ml} \mathrm{LPS}$, but it did not affect the phosphorylation of ERK (Fig. 6).

Effects of YCG063 on the activation of $N F-\kappa B$ in PA-LPS-stimulated ARPE-19 cells. The production of pro-inflammatory cytokines, chemokines and adhesion molecules is regulated by the transcription factor, $\mathrm{NF}-\kappa \mathrm{B}$, upon PA-LPS stimulation. Therefore, to investigate the mechanism through which YCG063 affects the expression of IL-6, IL-8, MCP-1 and ICAM-1, we examined the effects of YCG063 on NF- $\kappa$ B activation. We found that YCG063 inhibited the LPS-induced expression of NF- $\mathrm{B}$ p 65 in the nuclei (Fig. 7A). We then investigated the effects of YCG063 


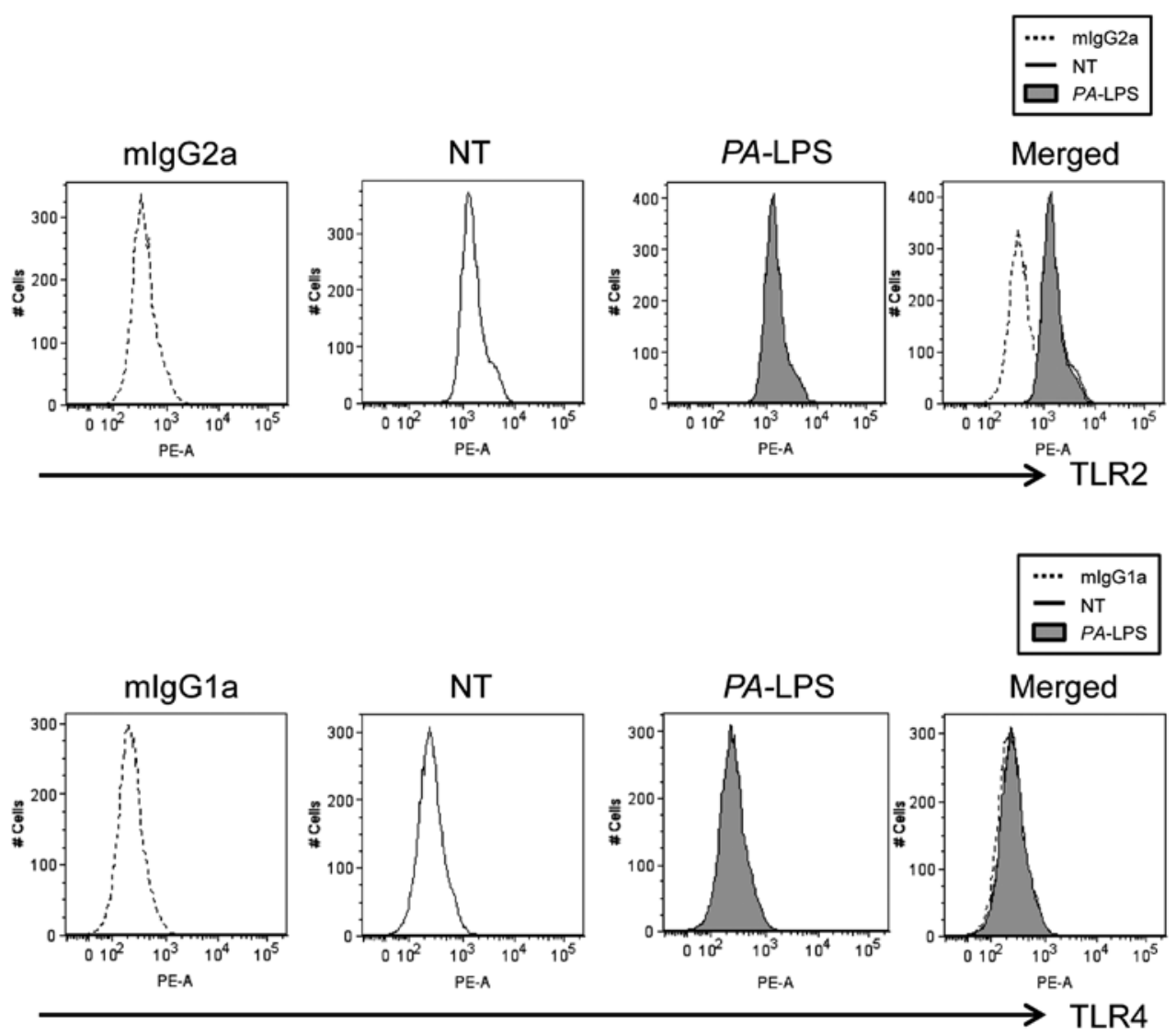

Figure 4. Expression of Toll-like receptors (TLRs) in Pseudomonas aeruginosa lipopolysaccharide (PA-LPS)-stimulated human adult retinal pigment epithelial cells (ARPE-19 cells). ARPE-19 cells were stimulated with PA-LPS $(10 \mu \mathrm{g} / \mathrm{ml})$ or were left unstimulated. FACS analysis using specific antibodies against TLR2 and TLR4 were performed. mIgG2a and mIgG1a, isotype control; NT, no treatment (medium).
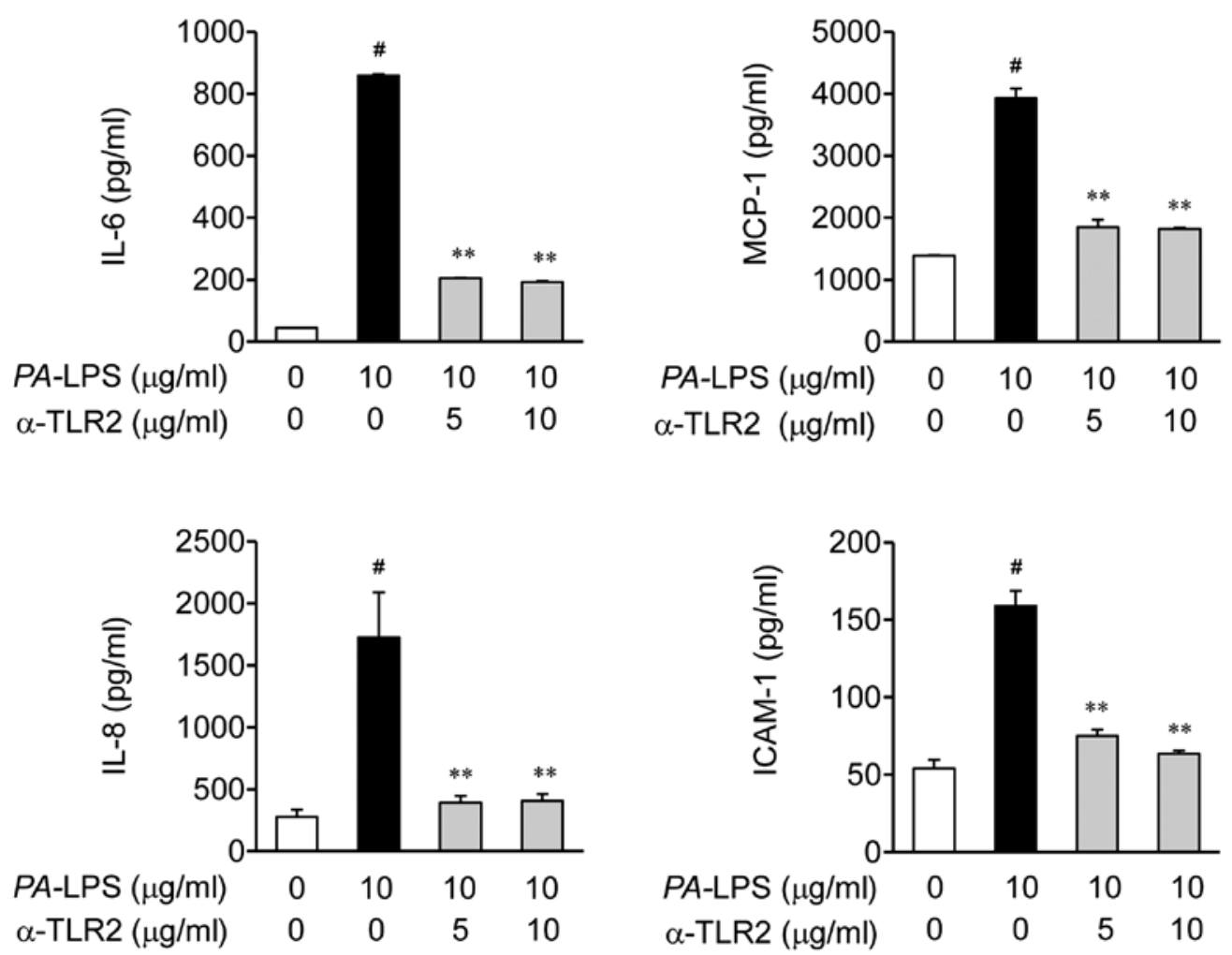

Figure 5. Toll-like receptor (TLR)2 is involved in the Pseudomonas aeruginosa lipopolysaccharide (PA-LPS)-induced expression of interleukin (IL)-6, IL-8, monocyte chemoattractant protein-1 (MCP-1) and intracellular adhesion molecule-1 (ICAM-1) in human adult retinal pigment epithelial cells (ARPE-19 cells). The ARPE-19 cells were pre-treated with antibody against TLR2 for $1 \mathrm{~h}$, followed by stimulation with PA-LPS (10 $\mu \mathrm{g} / \mathrm{ml})$ for $24 \mathrm{~h}$. The secretion of IL-6, IL-8, MCP-1 and ICAM-1 in the culture medium was measured by ELISA. 


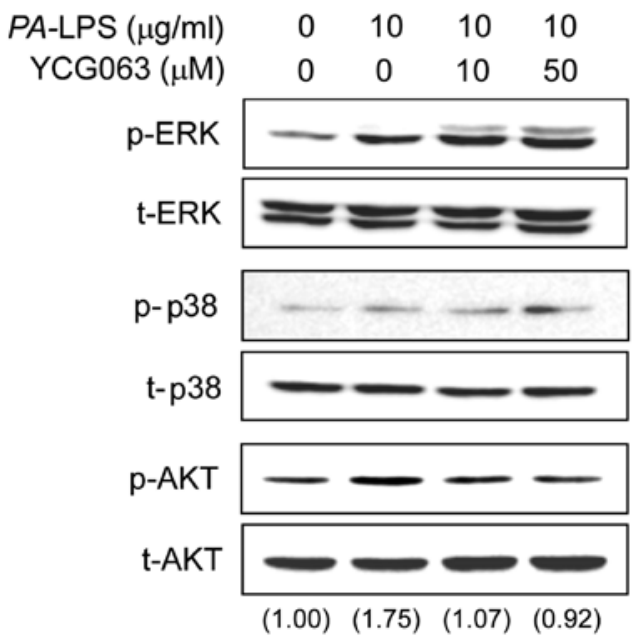

Figure 6. Effect of YCG063 on the Pseudomonas aeruginosa lipopolysaccharide (PA-LPS)-induced phosphorylation of ERK1/2, p38 MAPK and AKT in ARPE-19 cells. The human adult retinal pigment epithelial cells (ARPE-19 cells) were treated with the indicated concentrations of YCG063 for $2 \mathrm{~h}$ prior to stimulation with $P A$-LPS $(10 \mu \mathrm{g} / \mathrm{ml})$ for $30 \mathrm{~min}$. Cell extracts were then prepared and subjected to western blot analysis with antibodies specific for the phosphorylated forms of ERK1/2, p38 and AKT. The results are representative of 3 independent experiments. Control, untreated cells.

on the DNA-binding activity of NF- $\kappa$ B by EMSA (Fig. 7B). Stimulation with $P A$-LPS caused a significant increase in the DNA-binding activity of NF- $\mathrm{BB}$, whereas pre-treatment with YCG063 markedly reduced the $P A$-LPS-induced DNA-binding activity of NF-KB (Fig. 7B). Moreover, the upregulation in the expression of NF- $\mathrm{KB}$ p65 was significantly inhibited by treatment with LY294002 (an inhibitor of AKT; Fig. 7C).

Effects of NF- $\kappa B$ inhibitors (BAY 11-7082 and parthenolide) on the expression of $I L-6, I L-8, M C P-1$ and ICAM- 1 in PA-LPS-stimulated ARPE-19 cells. Based on the results shown in Fig. 7, we investigated whether BAY 11-7082 and parthenolide can suppress the LPS-induced expression of inflammatory mediators (Fig. 8). BAY 11-7082 and parthenolide inhibited the expression of IL-6, IL-8, MCP-1 and ICAM-1 in the LPS-stimulated ARPE-19 cells (Fig. 8).

Generation of ROS in PA-LPS-stimulated ARPE-19 cells. Since the production of inflammatory mediators in LPS-induced inflammation is associated with the generation of ROS (25), we investigated whether PA-LPS induces ROS generation. As shown by DCFDA staining, the intracellular levels of ROS did not increase following stimulation of the cells with $10 \mu \mathrm{g} / \mathrm{ml} P A$-LPS compared the untreated cells (basal levels; Fig. 9A). Additionally, according to the results of flow cytometry, the levels of two types of mitochondrial ROS (superoxide and hydrogen peroxide) did not increase following stimulation with $10 \mu \mathrm{g} / \mathrm{ml} P A$-LPS compared to the untreated cells (basal level; Fig. 9B).

\section{Discussion}

Recurrent endogenous endophthalmitis due to infection by $P A$ has been previously reported (5). In the present study, we investigated the anti-inflammatory effects of YCG063 (a wellknown ROS inhibitor) on the production of the inflammatory
A

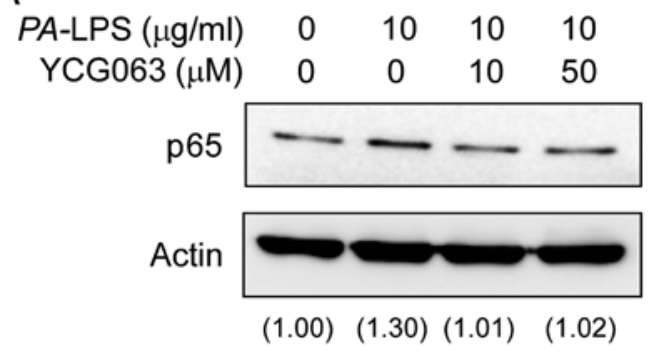

$\mathrm{B}$

$\begin{array}{rccccc}\text { PA-LPS }(\mu \mathrm{g} / \mathrm{ml}) & 0 & 10 & 10 & 10 & 10 \\ \text { YCG063 }(\mu \mathrm{M}) & 0 & 0 & 10 & 50 & 0 \\ \text { Cold-אB }(100 \mathrm{x}) & - & - & - & - & +\end{array}$

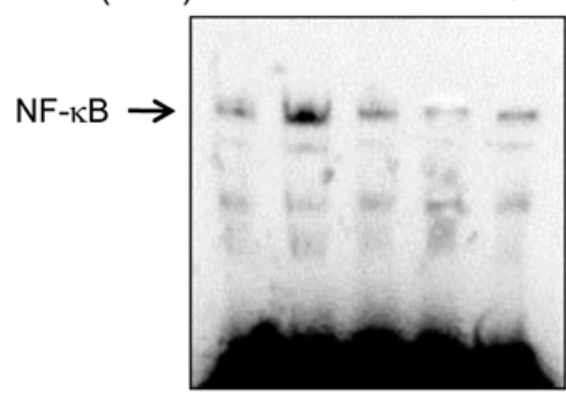

C PA-LPS $(\mu \mathrm{g} / \mathrm{ml}) \quad 0 \quad 10 \quad 10$ LY294002 $(\mu \mathrm{M}) \quad 0 \quad 0 \quad 30$

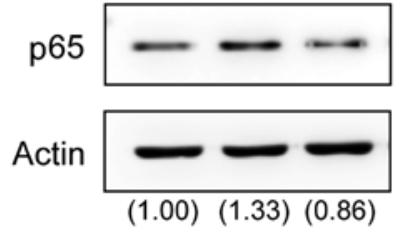

Figure 7. Effect of YCG063 on the translocation and binding of nuclear factor- $\kappa \mathrm{B}(\mathrm{NF}-\kappa \mathrm{B})$ into the nuclei of Pseudomonas aeruginosa lipopolysaccharide (PA-LPS)-stimulated ARPE-19 cells. The human adult retinal pigment epithelial cells (ARPE-19 cells) were pre-treated with YCG063 (10 or $50 \mu \mathrm{M}$ ) for $2 \mathrm{~h}$ and were then stimulated with $P A$-LPS for $1 \mathrm{~h}$. (A) Nuclear extracts were prepared as described in the Materials and methods and evaluated for NF- $\mathrm{B}$ by western blot analysis. (B) Nuclear protein extracts were prepared and analyzed for the DNA binding activity of NF- $\kappa \mathrm{B}$ using EMSA. Cold $-\kappa B$, unlabeled NF- $\kappa$ B probe. (C) The ARPE-19 cells were treated with $30 \mu \mathrm{M}$ of LY294002 for $2 \mathrm{~h}$ prior to stimulation with PA-LPS for $30 \mathrm{~min}$. Nuclear extracts were prepared and evaluated for NF- $\kappa$ B by western blot analysis. Control, untreated cells.

mediators, IL-6, IL-8, MCP-1 and ICAM-1, induced by PA-LPS in ARPE-19 cells.

The infiltration of PMNs in the eye is an important pathological process observed in intraocular infections, such as bacterial endophthalmitis (26). Inflammatory mediators induce increased PMN infiltration to the infected eye and, subsequently, severe retinal damage. The inflammatory mediators are expressed in response to Gram-negative bacterial infections and are mediated by bacterially derived products, such as LPS (27). In this study, we first investigated the inhibitory effects of YCG063 on the PA-LPS-induced production of IL-6, IL-8, MCP-1 and ICAM-1 in ARPE-19 cells. The levels of proinflammatory cytokines and chemokines in RPE cells suggest a role of this cell type in the clinical presentation of bacterial endophthalmitis. To the best of our knowledge, although 

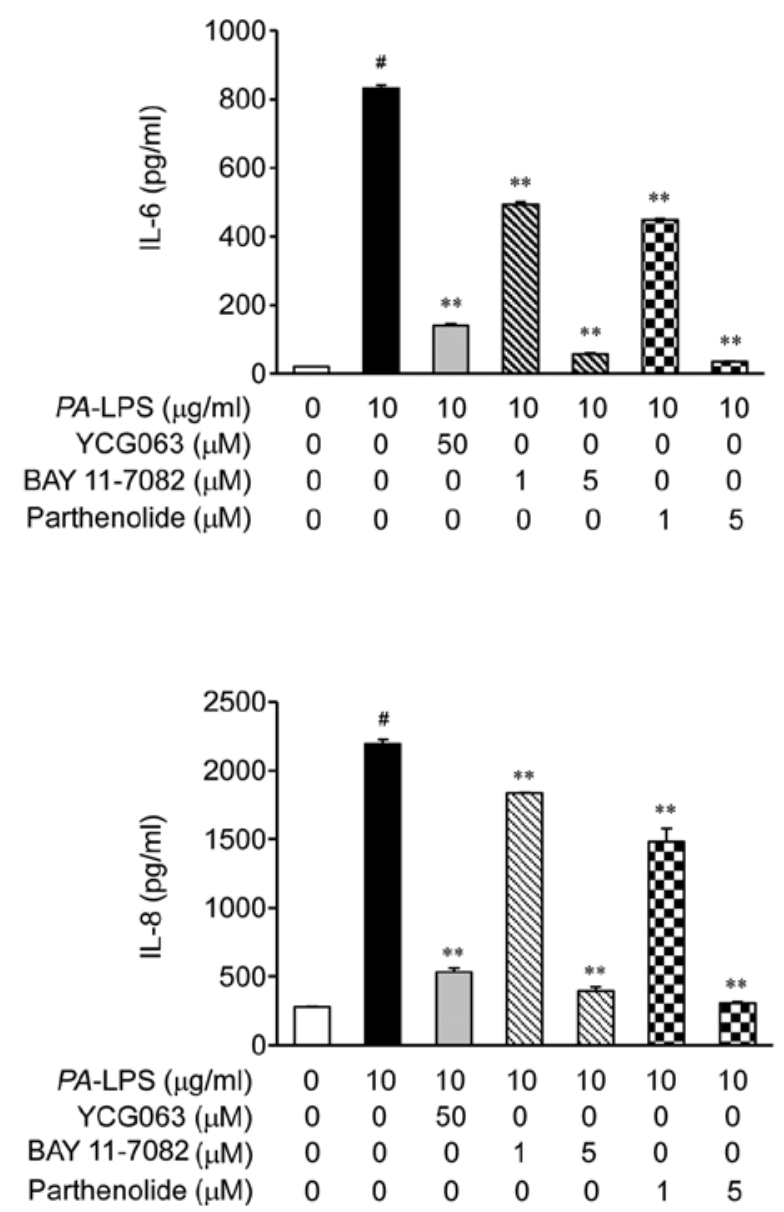
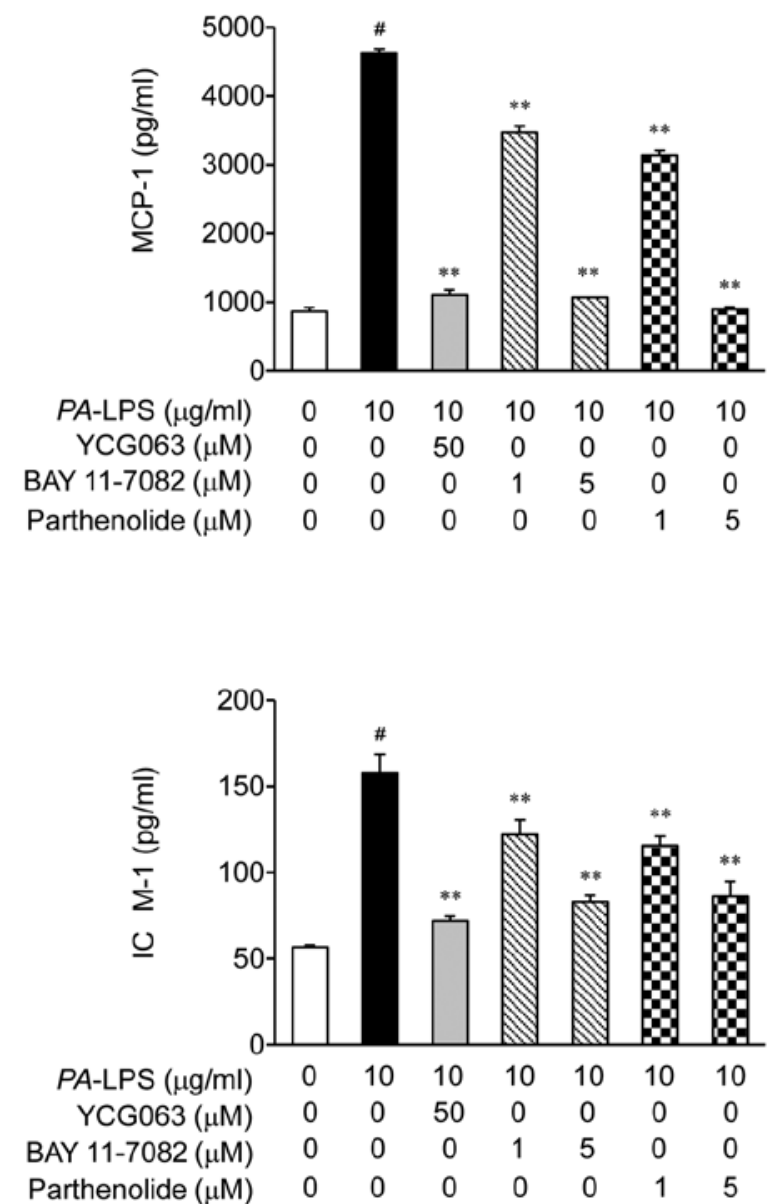

Figure 8. Effects of nuclear factor- $\kappa \mathrm{B}(\mathrm{NF}-\kappa \mathrm{B})$ inhibitors on the production of interleukin (IL)-6, IL-8, monocyte chemoattractant protein-1 (MCP-1) and intracellular adhesion molecule-1 (ICAM-1) in Pseudomonas aeruginosa lipopolysaccharide (PA-LPS)-stimulated human adult retinal pigment epithelial cells (ARPE-19 cells). The protein levels of IL-6, IL-8, MCP-1 and ICAM-1 were determined by ELISA. The cells were treated with BAY 11-7082 (1 and $5 \mu$ M) and parthenolide $(1$ and $5 \mu \mathrm{M})$ for $2 \mathrm{~h}$ prior to LPS stimulation for $24 \mathrm{~h}$. Each bar represents the mean \pm SD from 3 independent experiments. ${ }^{\#} \mathrm{p}<0.05$ compared with the untreated group. ${ }^{* *} \mathrm{p}<0.01$ compared with the LPS-stimulated values.

YCG063 has been investigated as an anti-angiogenic small molecule (24), a possible role in ocular inflammation has not been addressed. In this study, YCG063 significantly inhibited the LPS-induced expression of IL-6, IL-8, MCP-1 and ICAM-1 at concentrations of YCG063 that were not cytotoxic to the ARPE-19 cells (Fig. 3). Thus, the inhibition of the production of potent inflammatory mediators by pre-treatment with YCG063 may be an effective therapeutic approach with which to prevent retinal damage.

We also investigated which TLR members are involved in the inflammatory response induced by PA-LPS stimulation. The TLR family members are associated with the innate immune response and recognize specific pathogen-associated molecular patterns (PAMPs) expressed by different groups of infectious microbes (28). Ultimately, a cascade of intracellular signaling reactions occur and this leads to the activation of DNA binding proteins that, in turn, promote the transcription of pro-inflammatory genes $(29,30)$. They constitute as the first line of defense against invading pathogens and play a significant role in inflammation, immune cell regulation, survival and proliferation. In this study, we found that TLR2 was upregulated in the PA-LPS-stimulated ARPE-19 cells, whereas TLR4 was not (Fig. 4). To confirm this response, we investigated whether inflammatory mediators are inhibited by blocking TLR2 with an antibody against TLR2. The TLR2 antibody significantly inhibited the production of IL-6, IL-8, MCP-1 and ICAM-1 (Fig. 5). Our findings demonstrated that $P A$-LPS predominantly enhanced TLR2 expression in the ARPE-19 cells, leading to the increased production of IL-6, IL-8, MCP-1 and ICAM-1. The blockade of TLR4 did not have an inhibitory effect on the PA-LPS-stimulated ARPE-19 cells (data not shown).

Since YCG063 inhibited the expression of the inflammatory mediators in the PA-LPS-stimulated ARPE-19 cells, we further explored the regulatory mechanisms of YCG063 involved in this process (Fig. 6). The MAPK and AKT pathways are important downstream pathways of LPS/TLR signaling (31). To elucidate the regulatory mechanisms of YCG063 involved in these processes, we investigated whether the AKT and MAPK signaling pathways are involved in regulating the inflammatory mediators. Our findings demonstrated that ERK and AKT, but not p38 MAPK, were involved in the inflammatory responses of the PA-LPS-stimulated ARPE-19 cells. Therefore, the activation of the ERK and AKT pathways plays an important role in the $P A$-LPS-induced increase in the production of inflammatory mediators in ARPE-19 cells. However, our results demonstrated that YCG063 inhibited the PA-LPS-induced phosphorylation of AKT. Therefore, we demonstrated the blockage of the 


\section{Total ROS ( DCFDA)}

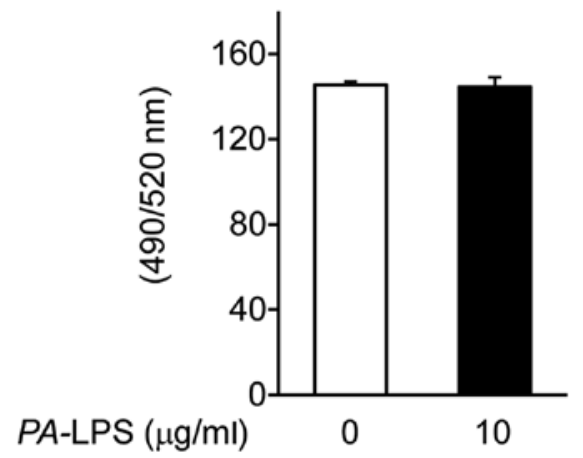

B
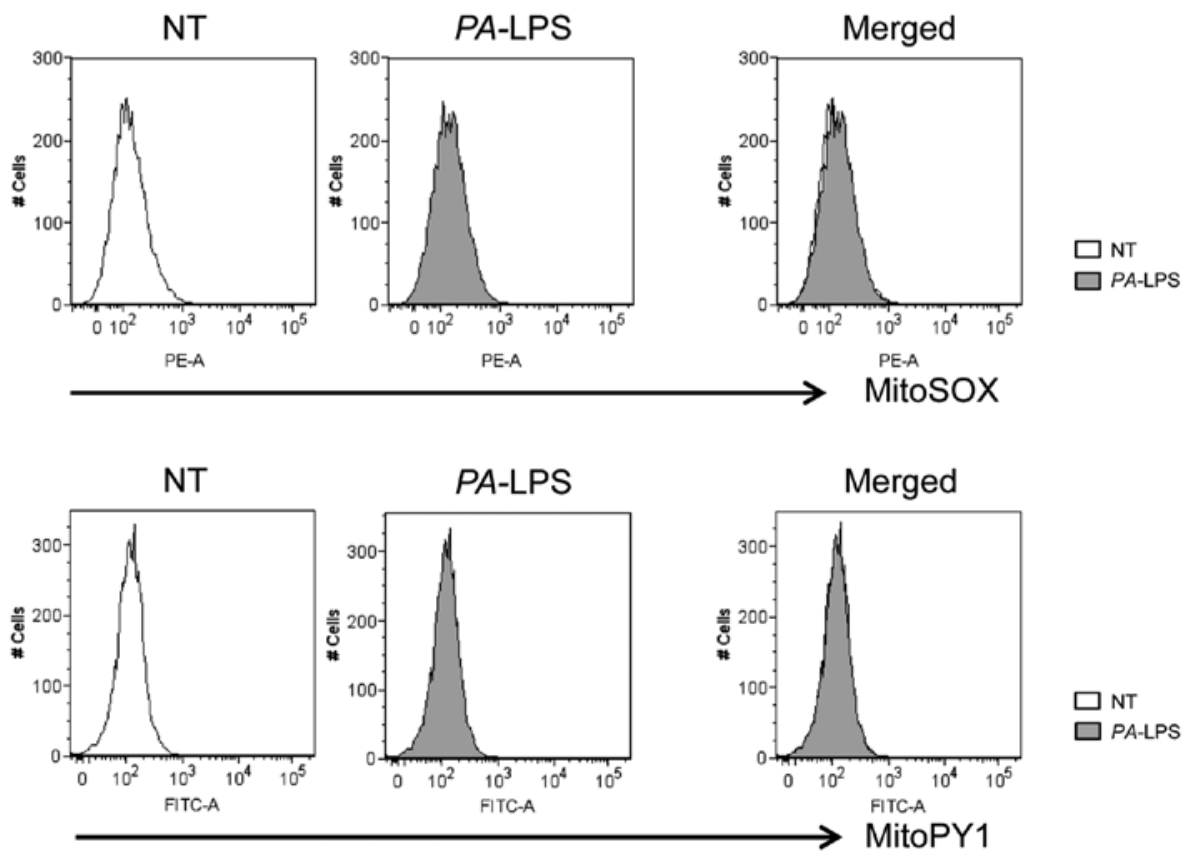

Figure 9. The Pseudomonas aeruginosa lipopolysaccharide (PA-LPS)-induced production of pro-inflammatory cytokines involves an reactive oxygen species (ROS)-independent mechanism in human adult retinal pigment epithelial cells (ARPE-19 cells). (A) The cells were stimulated with $10 \mu \mathrm{g} / \mathrm{ml} P A$-LPS for $5 \mathrm{~h}$. The intracellular ROS levels were measured using a fluorescence microplate reader. (B) ARPE-19 cells were stimulated with PA-LPS (10 $\mu \mathrm{g} / \mathrm{ml})$ for $24 \mathrm{~h}$. The cells were resuspended, and their mitochondrial ROS levels were measured by flow cytometry. The experiments are representative of 3 independent experiments, all of which displayed similar results. NT, no treatment.

$P A$-LPS-induced phosphorylation of AKT by YCG063 in ARPE-19 cells. To further elucidate the regulatory mechanisms of YCG063, we investigated whether the NF- $\mathrm{B}$ signaling pathway is involved in this process. $\mathrm{NF}-\kappa \mathrm{B}$ is known to be a pleiotropic regulator of various genes involved in the production of a number of pro-inflammatory cytokines and enzymes related to the inflammatory process (32). Due to its key role in several pathological conditions, $\mathrm{NF}-\kappa \mathrm{B}$ is a major drug target in a variety of diseases (33). Our results revealed that the LPS-induced activation of NF- $\mathrm{B}$ p 65 was completely abolished by pretreatment of the cells with YCG063. Therefore, the inhibition of the NF- $\kappa \mathrm{B}$ signaling pathway in the ARPE-19 cells by YCG063 may result in the downregulation of inflammatory responses. The AKT-dependent activation of NF- $\kappa \mathrm{B}$ has been described in a variety of cell types stimulated with platelet-derived growth factor, TNF- $\alpha$, IL-1, as well as other agonists (34). We found that the inhibition of AKT by LY-294002 blocked the translocation of NF- $\kappa$ B (Fig. 7C). Subsequently, we investigated whether the $\mathrm{NF}-\kappa \mathrm{B}$ inhibitors (BAY 11-7082 and parthenolide) are involved in regulating these inflammatory mediators. The NF- $\kappa \mathrm{B}$ inhibitors significantly suppressed the production of the IL-6, IL-8, MCP-1 and ICAM-1 proteins (Fig. 8). Taken together, these results suggest that the activation of $\mathrm{AKT}$ and $\mathrm{NF}-\kappa \mathrm{B}$, but not that of MAPKs, is involved in the inhibitory effects of YCG063 on the PA-LPS-induced production of IL-6, IL-8, MCP-1 and ICAM-1.

In a previous study, ROS were generated with $P A$-LPS in human adenocarcinoma alveolar basal epithelial cells (A549 cells) (35). In order to observe whether they contribute to the production of inflammatory mediators, we investigated whether ROS are generated in ARPE-19 cells by stimulation with PA-LPS (Fig. 9). The results revealed that the levels of ROS were not enhanced in the PA-LPS-stimulated ARPE-19 cells compared to the untreated cells. Thus, our findings 
suggest that the production of pro-inflammatory cytokines and chemokines triggered by $P A$-LPS is accomplished through ROS-independent mechanisms.

In conclusion, in this study, we demonstrated that PA-LPS induced IL-6, IL-8, MCP-1 and ICAM-1 expression in the ARPE-19 cells. Pre-treatment with YCG063 inhibited the expression of IL-6, IL-8, MCP-1 and ICAM-1 in the PA-LPSstimulated ARPE-19 cells. This inhibition by YCG063 may be the result of the suppression of the phosphorylation of AKT, as well as the inhibition of the activation of NF- $\kappa \mathrm{B}$ in modulating TLR2-induced inflammation. We also demonstrated that ROS are not involved in these inflammatory responses induced by $P A$-LPS. Based on our results, the inhibitory effects of YCG063 on inflammatory reactions may be considered as an effective therapeutic strategy for the treatment of bacterial endophthalmitis.

\section{Acknowledgements}

The present study was supported by a grant from the Korea Healthcare Technology R\&D Project, Ministry of Health and Welfare and Family Affairs, Republic of Korea (HI12C0005).

\section{References}

1. Bertino JS Jr: Impact of antibiotic resistance in the management of ocular infections: The role of current and future antibiotics. Clin Ophthalmol 3: 507-521, 2009.

2. Al-Amri MS: Endogenous endophthalmitis secondary to pyogenic liver abscess. Int J Diabet Mellitus 2: 646-646, 2010.

3. Callegan MC, Engelbert M, Parke DW II, Jett BD and Gilmore MS: Bacterial endophthalmitis: Epidemiology, therapeutics, and bacterium-host interactions. Clin Microbiol Rev 15: 111-124, 2002

4. Obritsch MD, Fish DN, MacLaren R and Jung R: Nosocomial infections due to multidrug-resistant Pseudomonas aeruginosa: Epidemiology and treatment options. Pharmacotherapy 25: 1353-1364, 2005.

5. Eifrig CW, Scott IU, Flynn HW Jr and Miller D: Endophthalmitis caused by Pseudomonas aeruginosa. Ophthalmology 110: 1714-1717, 2003.

6. Ren Y, Xie Y, Jiang G, Fan J, Yeung J, Li W, Tam PK and Savill J: Apoptotic cells protect mice against lipopolysaccharide-induced shock. J Immunol 180: 4978-4985, 2008.

7. Vallejo-Garcia JL, Asencio-Duran M, Pastora-Salvador N, Vinciguerra P and Romano MR: Role of inflammation in endophthalmitis. Mediators Inflamm 2012: 196094, 2012.

8. Campochiaro PA: Ocular neovascularization. J Mol Med Berl 91: 311-321, 2013.

9. Bonilha VL: Age and disease-related structural changes in the retinal pigment epithelium. Clin Ophthalmol 2: 413-424, 2008.

10. Arranz-Valsero I, Schulze U, Contreras-Ruiz L, García-Posadas L, López-García A, Paulsen F and Diebold Y: Involvement of corneal epithelial cells in the Th17 response in an in vitro bacterial inflammation model. Mol Vis 19: 85-99, 2013.

11. Gallucci RM, Simeonova PP, Matheson JM, Kommineni C, Guriel JL, Sugawara T and Luster MI: Impaired cutaneous wound healing in interleukin-6-deficient and immunosuppressed mice. FASEB J 14: 2525-2531, 2000.

12. Gallucci RM, Sugawara T, Yucesoy B, Berryann K, Simeonova PP, Matheson JM and Luster MI: Interleukin-6 treatment augments cutaneous wound healing in immunosuppressed mice. J Interferon Cytokine Res 21: 603-609, 2001.

13. Biswas PS, Banerjee K, Kinchington PR and Rouse BT: Involvement of IL-6 in the paracrine production of VEGF in ocular HSV-1 infection. Exp Eye Res 82: 46-54, 2006.

14. De Vos AF, Hoekzema R and Kijlstra A: Cytokines and uveitis, a review. Curr Eye Res 11: 581-597, 1992.
15. Fenton RR, Molesworth-Kenyon S, Oakes JE and Lausch RN: Linkage of IL-6 with neutrophil chemoattractant expression in virus-induced ocular inflammation. Invest Ophthalmol Vis Sci 43: 737-743, 2002.

16. Cole N, Bao S, Willcox M and Husband AJ: Expression of interleukin-6 in the cornea in response to infection with different strains of Pseudomonas aeruginosa. Infect Immun 67: 2497-2502, 1999.

17. Youker K, Smith CW, Anderson DC, Miller D, Michael LH, Rossen RD and Entman ML: Neutrophil adherence to isolated adult cardiac myocytes. Induction by cardiac lymph collected during ischemia and reperfusion. J Clin Invest 89: 602-609, 1992.

18. Liu Y, Shaw SK, Ma S, Yang L, Luscinskas FW and Parkos CA: Regulation of leukocyte transmigration: Cell surface interactions and signaling events. J Immunol 172: 7-13, 2004.

19. Tacke F and Randolph GJ: Migratory fate and differentiation of blood monocyte subsets. Immunobiology 211: 609-618, 2006

20. Van Riper G, Siciliano S, Fischer PA, Meurer R, Springer MS and Rosen H: Characterization and species distribution of high affinity GTP-coupled receptors for human rantes and monocyte chemoattractant protein 1. J Exp Med 177: 851-856, 1993.

21. Carr MW, Roth SJ, Luther E, Rose SS and Springer TA: Monocyte chemoattractant protein 1 acts as a T-lymphocyte chemoattractant. Proc Natl Acad Sci USA 91: 3652-3656, 1994.

22. Kawashima A, Suzuki T, Nishihara F, Kobayashi T, Takaku Y, Nakagome K, Soma T, Hagiwara K, Kanazawa M and Nagata M: Effect of formoterol on eosinophil trans-basement membrane migration induced by interleukin-8-stimulated neutrophils.Int Arch Allergy Immunol 161 (Suppl 2): 10-15, 2013.

23. Chen YJ, Huang YS, Chen JT, Chen YH, Tai MC, Chen CL and Liang CM: Protective effects of glucosamine on oxidativestress and ischemia/reperfusion-induced retinal injury. Invest Ophthalmol Vis Sci 56: 1506-1516, 2015.

24. Kim KH, Park JY, Jung HJ and Kwon HJ: Identification and biological activities of a new antiangiogenic small molecule that suppresses mitochondrial reactive oxygen species. Biochem Biophys Res Commun 404: 541-545, 2011.

25. Meng Z, Yan C, Deng Q, Gao DF and Niu XL: Curcumin inhibits LPS-induced inflammation in rat vascular smooth muscle cells in vitro via ROS-relative TLR4-MAPK/NF- $\kappa$ B pathways. Acta Pharmacol Sin 34: 901-911, 2013.

26. Novosad BD, Astley RA and Callegan MC: Role of Toll-like receptor (TLR) 2 in experimental Bacillus cereus endophthalmitis. PLoS One 6: e28619, 2011.

27. Schwarz JM and Bilbo SD: LPS elicits a much larger and broader inflammatory response than Escherichia coli infection within the hippocampus of neonatal rats. Neurosci Lett 497: 110-115, 2011.

28. Aderem A and Ulevitch RJ: Toll-like receptors in the induction of the innate immune response. Nature 406: 782-787, 2000.

29. Akira S and Takeda K: Toll-like receptor signalling. Nat Rev Immunol 4: 499-511, 2004.

30. Vogel SN, Fitzgerald KA and Fenton MJ: TLRs: Differential adapter utilization by toll-like receptors mediates TLR-specific patterns of gene expression. Mol Interv 3: 466-477, 2003.

31. McGuire VA, Gray A, Monk CE, Santos SG, Lee K, Aubareda A, Crowe J, Ronkina N, Schwermann J, Batty IH, et al: Cross talk between the Akt and p38 $\alpha$ pathways in macrophages downstream of Toll-like receptor signaling. Mol Cell Biol 33: 4152-4165, 2013.

32. Jung WK, Lee DY, Park C, et al: Cilostazol is anti-inflammatory in BV2 microglial cells by inactivating nuclear factor-kappaB and inhibiting mitogen-activated protein kinases. Br J Pharmacol 159: 1274-1285, 2010.

33. Dabek J, Kułach A and Gasior Z: Nuclear factor kappa-light-chainenhancer of activated B cells $(\mathrm{NF}-\kappa \mathrm{B})$ : a new potential therapeutic target in atherosclerosis? Pharmacol Rep 62: 778-783, 2010.

34. Chen BC, Wu WT, Ho FM and Lin WW: Inhibition of interleukin-1beta-induced NF-kappa B activation by calcium/calmodulin-dependent protein kinase kinase occurs through Akt activation associated with interleukin-1 receptor-associated kinase phosphorylation and uncoupling of MyD88. J Biol Chem 277: 24169-24179, 2002.

35. Li W, Yan F, Zhou H, Lin X, Wu Y, Chen C, Zhou N, Chen Z, Li JD and Shen H: P. aeruginosa lipopolysaccharide-induced MUC5AC and CLCA3 expression is partly through Duox 1 in vitro and in vivo. PLoS One 8: e63945, 2013. 